\title{
Dissociative Experiences in opioid-dependent patients on substitution therapy
}

\author{
A. Zwierzchowska1 ${ }^{1}$ E.J. Gorzelańczyk², P. Walecki ${ }^{1}$
}

1 Jagiellonian University Medical College, Faculty of Medicine, Krakow, Poland

2 Kazimierz Wielki University, Laboratory of Cognitive Science Institute of Philosophy, Bydgoszcz, Poland.

\section{OBJECTIVES}

The aim of the study is to assess the occurrence of dissociative experiences in addicts (not under the influence of psychoactive substances) in comparison to healthy ones.

The Dissociative Experiences are variety including both pathological dissociative experiences, and normal dissociative experience. Dissociation can be defined as a separation of functions, which are normally integrated: conciousness, memory, identity, perception; disengagement of intentional and automatic processes. We are defining the process of dissociation as a disruption in the usually integrated function of consciousness, memory, identity, or perception of the environment (definition according to DSM-IV), dissection of intentional and automatic processes. In the clinical literature, a distinction is often made between dissociative states and dissociative traits. Whereas state dissociation is viewed as a transient symptom (e.g., acute dissociation during a traumatic event), trait dissociation is viewed as an integral aspect of personality. As dissociative symptoms are prevalent in both nonclinical and clinical populations, dissociation has commonly been conceptualized as ranging on a continuum, from nonpathological manifestations of daydreaming to more severe disturbances typical of dissociative disorders. These disorders include dissociative amnesia (extensive forgetting typically associated with highly aversive events), dissociative fugue (short-lived reversible amnesia for personal identity, involving unplanned travel or wandering), depersonalization disorder (DPD; feeling as though one is an outside observer of one's body and feeling like an automaton or like living in a dream or a movie; an experience technically referred to as derealization), and dissociative identity disorder (DID; experiencing two or more distinct identities that recurrently take control over one's behavior, APA, 2000).

METHODS

Eighteen opioid-addicted patients from Med-Seven Substitution Treatment Clinic in Bydgoszcz and twenty four healthy people were examined. The Dissociative Experience Scale (DES-II) and the NEO Five-Factor Personality Inventory (NEOFFI) was used.

\section{RESULTS}

The high level of dissociative experiences in people addicted to opioids was found. Opioid-addicted patients were significantly higher DES Score compared to healthy ones. On the basis of NEO-FFI results, it can be concluded that addicted patients has higher level of neuroticism compared to healthy ones and lower level of extraversion and conscientiousness compared to healthy ones. DES Score was positively correlated with the higher neuroticism level. DES Score was also negative correlated with lower level of extraversion and lower level of conscientiousness.

Opioid-addicted patients presented signifcantly $(p<0.001)$ :
- Higher DES-II
Score
$(22.21 \pm 13.01)$
compared to healthy ones $(4.99 \pm 2.63)$
- Higher level of neuroticism (7.20 \pm 1.94$)$ compared to healthy ones $(3.27 \pm 1.83)$
- Lower level of extraversion (3.70 2.15$)$ compared to healthy ones (6.14 2.29 )
- Lower level of conscientiousness $(4.00 \pm 1.89)$ compared to healthy ones $(6.59 \pm 1.84)$

DES Score was positively correlated $(p<0.05)$ with:

- higher neuroticism level

- lower level of extraversion

- lower level of conscientiousness

Neuroticism level was positively correlated (p>0.05) with:

- lower extraversion level

- lower conscientiousness level

\section{CONCLUSIONS}

The results are suggesting common mechanisms responsible for dissociative experiences and using psychoactive substances, also genesis of addiction. The outcomes are encouraging to continue the researches to determine mechanisms which are connecting dissociation and addiction, and also to define the biological factors responsible for this phenomenon. 